\title{
Adjuvant vs. salvage radiation therapy in men with high-risk features after radical prostatectomy: Survey of North American genitourinary expert radiation oncologists
}

\author{
Shearwood McClelland 3rd ${ }^{1}$; Kiri A. Sandler ${ }^{2}$ : Catherine Degnin ${ }^{3}$; Yiyi Chen ${ }^{3}$; Timur Mitin ${ }^{1}$ \\ ${ }^{1}$ Department of Radiation Medicine, Oregon Health and Science University, Portland, OR, United States; \\ ${ }^{2}$ Department of Radiation Oncology, University of California at Los Angeles, Los Angeles, CA, United States; \\ ${ }^{3}$ Biostatistics Shared Resource, Oregon Health and Science University, Portland, OR, United States
}

Cite as: Can Urol Assoc J 2018 October 15; Epub ahead of print. http://dx.doi.org/10.5489/cuaj.5470

Published online October 15, 2018

$* * *$

\begin{abstract}
Introduction: The management of patients with high-risk features after radical prostatectomy is controversial. Level 1 evidence demonstrates that adjuvant RT improves survival compared to no treatment; however, it may overtreat up to $30 \%$ of patients, as randomized clinical trials (RCTs) using salvage RT on observation arms failed to reveal a survival advantage of adjuvant RT. We, therefore, sought to determine the current view of adjuvant vs. salvage RT among North American genitourinary (GU) radiation oncology experts.

Methods: A survey was distributed to 88 practicing North American GU physicians serving on decision-making committees of cooperative group research organizations. Questions pertained to opinions regarding adjuvant vs. salvage RT for this patient population. Treatment recommendations were correlated with practice patterns using Fisher's exact test.

Results: Forty-two of 88 radiation oncologists completed the survey; 23 (54.8\%) recommended adjuvant RT and 19 (45.2\%) recommended salvage RT. Recommendation of active surveillance for Gleason 3+4 disease was a significant predictor of salvage RT recommendation $(p=0.034)$, and monthly patient volume approached significance for recommendation of adjuvant over salvage RT; those seeing < 15 patients/month trended towards recommending adjuvant over salvage RT ( $\mathrm{p}=0.062)$. No other demographic factors approached significance.

Conclusions: There is dramatic polarization among North American GU experts regarding optimal management of patients with high-risk features after radical prostatectomy. Ongoing RCTs will determine whether adjuvant RT improves survival over salvage RT. Until then, the almost 50/50 division seen from this analysis should encourage practicing clinicians discuss the ambiguity with their patients.
\end{abstract}




\section{Introduction}

Three randomized clinical trials have established the role of adjuvant radiation therapy. ${ }^{1-3}$ SWOG 8794 revealed a survival advantage when patients who received adjuvant RT were compared to patients who were followed clinically with no salvage RT option even in the setting of PSA failure. ${ }^{1}$ The other two trials - EORTC 22911 and ARO 96/02 - failed to reveal an overall survival advantage, likely due to the protocol stipulation of allowed or recommended salvage RT in men randomized to observation in case of biochemical failure. ${ }^{2-3}$ Moreover, all three trials have shown a 10 -year biochemical progression free survival rate of $26-41 \%$ in the observation arm, arguing that a third of patients with high risk features after radical prostatectomy will never develop biochemical failure and therefore would receive unnecessary overtreatment with pelvic radiotherapy. ${ }^{4-6}$ Two large modern randomized trials (RAVES, RADICALS) are under way to help physicians determine if adjuvant RT has any advantage over initial observation and early salvage RT, but until results are published, this topic remains highly controversial. ${ }^{7-8}$ We sought to determine the current view of adjuvant versus salvage RT among North American genitourinary (GU) radiation oncology experts due to their influence in shaping clinical trials and national guidelines.

\section{Methods}

\section{Survey design and deployment}

The survey was designed to assess the opinion of GU experts on the preferred management of a hypothetical patient with a high risk feature (extracapsular extension) following radical prostatectomy for prostate cancer - adjuvant RT or observation with early salvage RT only if PSA rises. A copy of the survey is available in the Appendix. The study was approved by the Institutional Review Board and electronically sent in November 2016 to 88 North American GU oncology physicians, who serve on cooperative group research organizations such as NRG Oncology. The survey was designed and hosted by Research Electronic Data Capture (REDCap). ${ }^{9}$

\section{Statistical analysis}

Based on responses, participants were categorized as supporters of either adjuvant RT or salvage RT for men with high risk features following radical prostatectomy. Treatment recommendations were correlated with practice patterns using Fisher's exact test.

\section{Results}

Forty-two of the 88 radiation oncologists completed the survey, of whom 23 (54.8\%) recommended adjuvant RT after radical prostatectomy; the remaining 19 (45.2\%) recommended observation with early salvage RT if PSA rises (Figure 1).

No demographic factors (years in practice, geographic location of residency, geographic location of practice, monthly patient volume, practice type) were found to correlate with treatment recommendation. When we analyzed for association with other treatment recommendations for men with prostate cancer, only recommendation of active surveillance for Gleason 3+4 disease 
was a significant predictor of recommending salvage RT following radical prostatectomy for disease with high-risk features $(p=0.034)$ (Table 1$)$. No other treatment recommendations (active surveillance recommendation for Gleason 6 disease, first choice treatment preference for lowrisk prostate cancer, brachytherapy boost for high risk disease, consideration of stereotactic body RT for oligometastatic disease, elective pelvic lymph node coverage, support for incorporation of advanced imaging modalities in standard practice) were significant. Monthly patient volume approached significance for recommendation of adjuvant RT over salvage RT - respondents who see fewer than 15 patients per month were more likely to endorse adjuvant RT over salvage RT $(p=0.062)$.

\section{Discussion}

Although biochemical control of prostate cancer with high-risk features following radical prostatectomy (extracapsular extension, seminal vesicle invasion, and/or positive surgical margins) has indisputably been shown to be improved by adjuvant RT in three randomized clinical trials, only one of these trials have shown an improvement in OS - when patients randomized to observation were not offered salvage RT in case of biochemical progression. ${ }^{1-6}$ The other two trials either recommended and stipulated salvage RT on observation arm, and failed to show a survival advantage to upfront intervention with adjuvant pelvic RT. Moreover, in all three trials a third of patients on observation arm never experienced biochemical failure on observation arms, despite having high risk features after radical prostatectomy. The 2017 National Comprehensive Cancer Network (NCCN) guidelines delineate indications for adjuvant RT as “pT3 disease, positive margin(s), Gleason score 8-10, or seminal vesicle involvement” and that "evidence supports offering adjuvant/salvage RT in most men with adverse pathologic features or detectable PSA and no evidence of disseminated disease". ${ }^{10}$

The results of our study indicate that for men with high risk features after radical prostatectomy, North American GU experts who are more likely to recommend salvage RT are also those who are more likely to recommend active surveillance for Gleason 3+4 disease. This intuitively makes sense, as physicians who are more comfortable with initiation observation of patients with intermediate risk prostate cancer [established by the recently published ProtecT randomized trial ${ }^{11}$ ] should also feel as comfortable with initial observation of men with high risk features after radical prostatectomy. Although no other demographic factor proved significant, the trend of experts seeing fewer than 15 patients/month being more likely to recommend adjuvant RT over salvage RT is interesting and deserves further investigation; perhaps highvolume experts are more likely to believe in salvage RT than their low-volume counterparts. It is our hope that ongoing phase III randomized clinical trials in this arena such as the Radiotherapy - Adjuvant versus Early Salvage (RAVES) and RADICALS trials will shed more light on adjuvant versus early salvage RT. ${ }^{7-8}$ Our study shares the limitations of the survey from which it is derived: a relatively small sample size, inability to capture a full range of options due to multiple choice format, and a lack of granularity in addressing the socioeconomic and racial demographic of patients, the latter of which may impact the applicability of randomized controlled trials comprised of inadequately low nonwhite patient participation. ${ }^{12-13}$ 


\section{Conclusion}

In conclusion, there is currently a nearly even split between radiation oncology experts in North America recommending adjuvant vs salvage RT for patients with high risk features after radical prostatectomy for prostate cancer. Ongoing large randomized trials will determine whether adjuvant therapy offers a survival advantage over salvage radiation therapy. Until then, the almost 50/50 division seen among leading GU experts according to this analysis should help practicing clinicians discuss the ambiguity with their patients. National care and reimbursement policies may also influence the accepted standard of care. 


\section{References}

1. Thompson IM Jr, Tangen CM, Paradelo J, et al. Adjuvant radiotherapy for pathologically advanced prostate cancer: a randomized clinical trial. JAMA. 2006;296:2329-2335.

2. Bolla M, van Poppel H, Collette L, et al. Postoperative radiotherapy after radical prostatectomy: a randomized controlled trial (EORTC trial 22911). Lancet. 2005;366:572-578.

3. Wiegel T, Bottke D, Steiner U, et al. Phase III postoperative adjuvant radiotherapy after radical prostatectomy compared with radical prostatectomy alone in pT3 prostate cancer with postoperative undetectable prostate-specific antigen: ARO 96-02/AUO AP 09/95. J Clin Oncol. 2009;27:2924-2930.

4. Thompson IM, Tangen CM, Paradelo J, et al. Adjuvant radiotherapy for pathologic T3N0M0 prostate cancer significantly reduces risk of metastases and improves survival: long-term followup of a randomized clinical trial. J Urol. 2009;181:956-962.

5. Bolla $\mathrm{M}$, van Poppel $\mathrm{H}$, Tombal B, et al. Postoperative radiotherapy after radical prostatectomy for high-risk prostate cancer: long-term results of a randomised controlled trial (EORTC trial 22911). Lancet. 2012;380:2018-2027.

6. Wiegel T, Bartkowiak D, Bottke D, et al. Prostate-specific antigen persistence after radical prostatectomy as a predictive factor of clinical relapse-free survival and overall survival: 10-year data of the ARO 96-02 trial. Int J Radiat Oncol Biol Phys. 2015;91:288294.

7. Pearse M, Fraser-Browne C, Davis ID, et al. A Phase III trial to investigate the timing of radiotherapy for prostate cancer with high-risk features: background and rationale of the Radiotherapy - Adjuvant Versus Early Salvage (RAVES) trial. BJU Int. 2014;113 Suppl 2:7-12.

8. Parker C, Sydes MR, Catton C, et al. Radiotherapy and androgen deprivation in combination after local surgery (RADICALS): a new Medical Research Council/National Cancer Institute of Canada phase III trial of adjuvant treatment after radical prostatectomy. BJU Int. 2007;99:1376-1379.

9. Harris PA, Taylor R, Thielke R, et al. Research electronic data capture (REDCap)--a metadata-driven methodology and workflow process for providing translational research informatics support. J Biomed Inform 2009;42:377-381.

10. National Comprehensive Cancer Network. NCCN Guidelines Version 2.2017, Prostate Cancer. https://www.nccn.org/professionals/physician_gls/pdf/prostate.pdf Accessed December 10, 2017.

11. Hamdy FC, Donovan JL, Lane JA, et al. 10-Year Outcomes after Monitoring, Surgery, or Radiotherapy for Localized Prostate Cancer. N Engl J Med. 2016;375:1415-1424.

12. McClelland S 3rd, Sandler KA, Degnin C, Chen Y, Mitin T. Active surveillance for low and Intermediate risk prostate cancer: opinions of North American genitourinary oncology expert radiation oncologists. Clin Genitourin Cancer. 2018;16:e323-e325.

13. McClelland S 3rd, Mitin TM. The danger of applying the ProtecT trial to minority populations. JAMA Oncol. 2018;4:291. 
Figures and Tables

Fig. 1. Default recommendation for men with high-risk features after radical prostatectomy for prostate cancer among North American genitourinary oncology expert radiation oncologists.

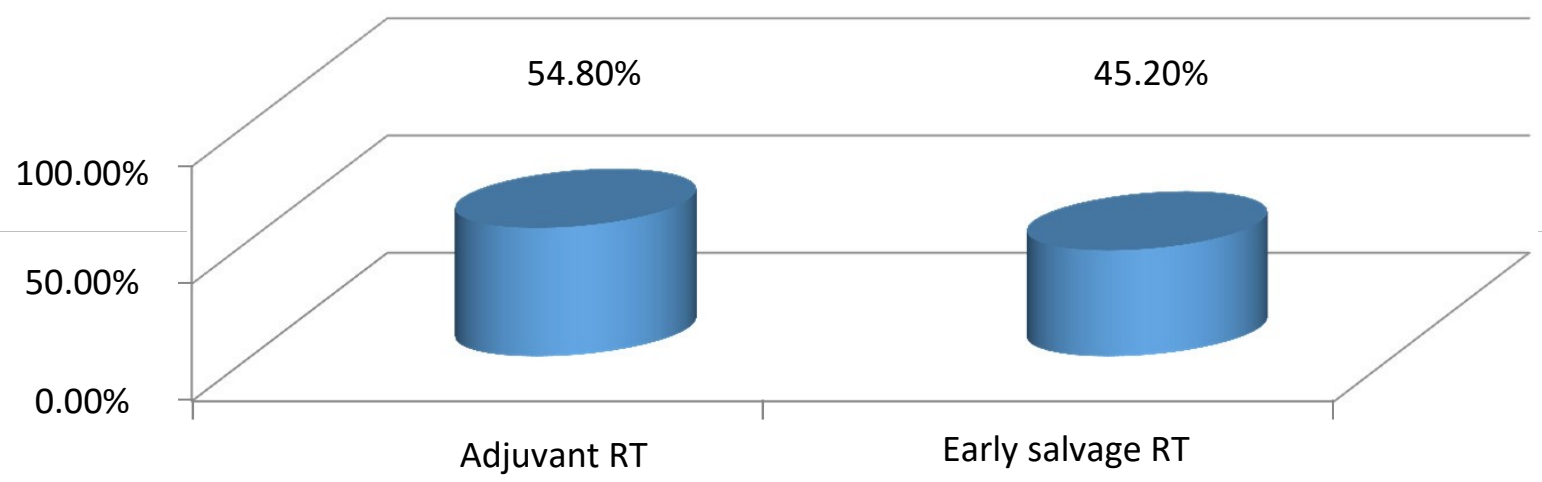




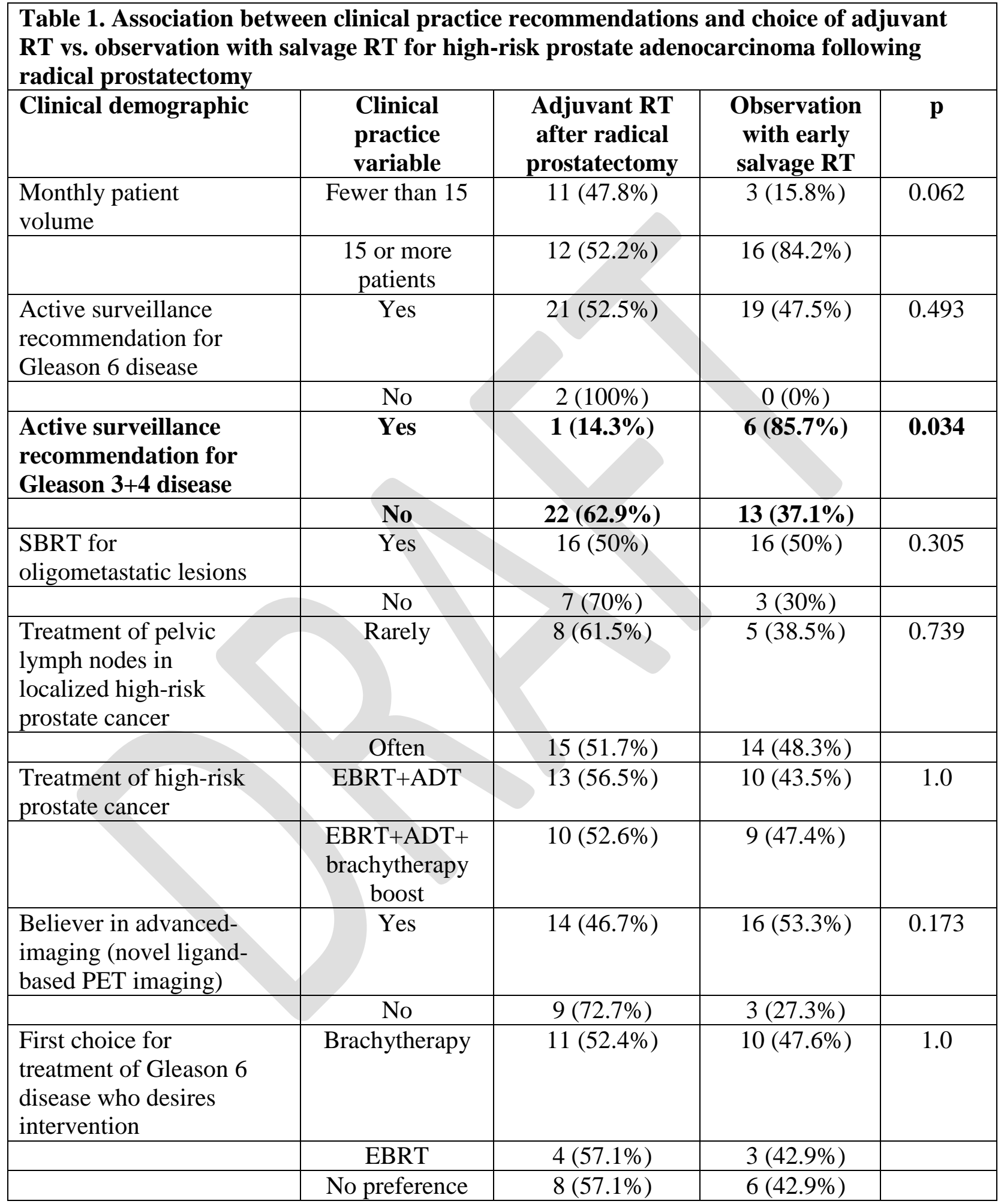

EBRT: external beam radiation therapy; PET: positron emission tomography; RT: radiation therapy; SBRT: stereotactic body radiation therapy. 